\title{
Mit sieben Kriterien zur Asthma-Diagnose
}

\begin{abstract}
Bei Patienten mit Atemwegsbeschwerden unklarer Genese lässt sich anhand einer Sieben-Punkte-Diagnostik klären, ob ein Asthma bronchiale vorliegt.
\end{abstract}

Die Global Initiative for Asthma (GINA) hat letztes Jahr ein Update ihrer Leitlinie zu Management und Prävention von Asthma veröffentlicht.

Um die Umsetzung im hausärztlichen Alltag zu erleichtern, haben Pneumologen um Dr. Ekkehard Beck, Rüdersdorf, die wichtigsten Schritte in einem einfachen Behandlungspfad zusammengefasst [1].

Dr. Martin Kohlhäufl, Klinik Schillerhöhe in Stuttgart, hat diesen beim Praxis Update in München vorgestellt. Wenn ein Patient mit Atemwegsbeschwerden unklarer Genese in die Praxis kommt, empfehlen die Experten eine SiebenPunkte-Diagnostik. Verdächtig auf ein Asthma bronchiale sind:

- Trockener Husten am Tag

- Aufwachen mit Husten in der Nacht/ am Morgen

- Giemen beim Atmen

- Brustenge/Atemnot

- Verstärkung der Symptome bei Virusinfektionen, feuchtkalter Luft sowie bei körperlicher Belastung Verlängertes Exspirium oder Giemen bei der Auskultation

- Eine FEV1 < 80\% vom Soll in der Spirometrie (nur bei positivem Auskultationsbefund empfohlen).
„Wenn von den sieben genannten Punkten mindestens drei erfüllt sind, weist das in Richtung Asthma und rechtfertigt die Einleitung einer Asthmatherapie“, sagte Kohlhäufl. Dazu soll der Patient, zunächst befristet auf vier Wochen, ein kurz wirksames Betamimetikum erhalten. „Wenn der Patient das mehr als dreimal pro Woche braucht, müssen Sie direkt in die Basistherapie mit einem niedrig dosierten inhalativen Kortikosteroid (ICS) einsteigen."

\section{Expertenmeinung gefragt}

Wenn Symptome nachts und am Morgen fehlen, rät Kohlhäufl, einen Pneumologen hinzuziehen, um andere Diagnosen abzuklären. Direkt an den Facharzt überwiesen werden sollten Patienten mit schwerer Symptomatik, einer FEV1 $<60 \%$ vom Soll und unveränderter Symptomatik trotz einer Therapie mit inhalativem Kortikosteroid plus lang wirksamem Betamimetikum. Als kontrolliert ist ein Asthma anzusehen, wenn die Patienten höchstens zweimal pro Woche tagsüber Symptome haben, nachts nicht wegen ihres Asthmas aufwachen, höchstens zweimal pro Woche ein Notfallspray anwenden und in ihren Aktivitäten nicht eingeschränkt sind.

(bs)

Atemwegs- und Lungenkrankheiten 2016; 42: 14-24

\section{ÜBRIGENS}

... verschlechtert sich Asthma bei Kindern, wenn bei ihnen zu Hause geraucht wird. Das zeigt sich besonders deutlich an der Häufigkeit stationärer Einweisungen.

Ann Allergy Asthma Immunol 2015; 115(5): 396-401.e2

... erkranken Kinder mit engem Kontakt zu Tieren seltener an Asthma. Das bestätigt eine Registerstudie mit Kindern, die im ersten Lebensjahr mit einem Hund oder auf dem Bauernhof gelebt hatten.

JAMA Pediatr 2015; 169: e153219

Punkte sammeln in der

Sommerakademie mit e.Med

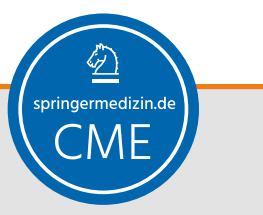

Auch 2016 haben wir in der Sommerakademie ausgewählte CME-Kurse für Ihre hausärztliche Fortbildung zusammengestellt.

Zum Thema "Asthma/COPD“ finden Sie unter www.springermedizin.de/sommerakademie aktuell den Kurs

Notfalldiagnostik und therapeutisches Management der akuten Dyspnoe"

Zur Teilnahme benötigen Sie das e.Med-Abo, mit dem Sie Zugang zu allen Inhalten auf Springer-Medizin.de erhalten - kombiniert mit einer gedruckten Fachzeitschrift Ihrer Wahl. Als e.Med-Abonnent haben Sie nicht nur Zugriff auf die Sommerakademie, Sie können das komplette CME-Kursangebot von SpringerMedizin.de nutzen. Hier finden Sie aktuell rund 550 CME-zertifizierte Fortbildungskurse aus allen medizinischen Fachrichtungen! Damit ist CME.SpringerMedizin.de Deutschlands erste Adresse für Online-CMEFortbildung.

Nutzen Sie die Sommerzeit, um CME-Punkte zu sammeln und um sich schnell und effizient fortzubilden. Testen Sie CME.SpringerMedizin.de 30 Tage lang kostenlos und unverbindlich mit dem e.Med-Abo: www.springermedizin.de/eMed 